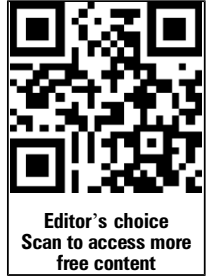

${ }^{1}$ Department for Health, University of Bath, Bath, UK ${ }^{2}$ Department of Sports Medicine, Rugby Football Union, Twickenham, UK ${ }^{3}$ Imperial College Healthcare NHS Trust, London, UK ${ }^{4}$ Colin Fuller Consultancy, Sutton Bonington, UK ${ }^{5}$ Karabati Limited, Nottingham, UK

Correspondence to Dr Keith A Stokes, Department for Health, University of Bath, Bath BA2 7AY, UK; k.stokes@bath.ac.uk

Accepted 10 September 2015 Published Online First 9 November 2015

\title{
Time loss injuries compromise team success in Elite Rugby Union: a 7-year prospective study
}

\author{
Sean Williams, ${ }^{1}$ Grant Trewartha, ${ }^{1}$ Simon P T Kemp, ${ }^{2}$ John H M Brooks, ${ }^{3}$ \\ Colin W Fuller, ${ }^{4}$ Aileen E Taylor, ${ }^{5}$ Matthew J Cross, ${ }^{1}$ Keith A Stokes ${ }^{1}$
}

\begin{abstract}
Background A negative association between injuries and team success has been demonstrated in professional football, but the nature of this association in elite Rugby Union teams is currently unclear.
\end{abstract}

Aim To assess the association between injury burden measures and team success outcomes within professional Rugby Union teams.

Methods A seven-season prospective cohort design was used to record all time-loss injuries incurred by English Premiership players. Associations between team success measures (league points tally and Eurorugby Club Ranking (ECR)) and injury measures (injury burden and injury days per team-match) were modelled, both within (changes from season to season) and between (differences averaged over all seasons) teams. Thresholds for the smallest worthwhile change in league points tally and ECR were 3 points and $2.6 \%$, respectively.

Results Data from a total of 1462 players within 15 Premiership teams were included in the analysis. We found clear negative associations between injury measures and team success (70-100\% likelihood), with the exception of between-team differences for injury days per team-match and ECR, which was unclear. $A$ reduction in injury burden of 42 days ( $90 \% \mathrm{Cl} 30$ to 70 ) per 1000 player hours (22\% of mean injury burden) was associated with the smallest worthwhile change in league points tally.

Conclusions Clear negative associations were found between injury measures and team success, and moderate reductions in injury burden may have worthwhile effects on competition outcomes for professional Rugby Union teams. These findings may be useful when communicating the value of injury prevention initiatives within this elite sport setting.

\section{INTRODUCTION}

Although success in team sports is dependent on a number of factors (eg, player skill, fitness, squad size, tactics and psychological factors), it has been argued that player durability may currently be an under-recognised component of team success. ${ }^{1}$ Player durability refers to a player's ability to tolerate the demands of their sport without incurring injuries, and thus remain available for selection. Injuries that result in time-loss from training and/or match-play may influence a team's chances of success via a number of mechanisms. For instance, a high team injury burden (injury incidence rate $x$ mean absence per injury) may prevent a coach from selecting the best players for a given match, while player absences from training sessions may disrupt a team's tactical preparations. ${ }^{2}$ There may also be negative psychological effects (for the injured player and/or the team) associated with injury incidents. ${ }^{3}$ In professional Rugby Union, injury incidence rates and the resulting absence of players from matches and training is high in comparison with some team sports, ${ }^{5}$ as such, the association between injuries and team success in this setting may be especially pertinent.

A 15 -season study involving one French professional football (soccer) team reported no significant relationship between final league position and injury incidence rates. ${ }^{6}$ Conversely, a multiteam prospective cohort study involving Qatari firstdivision clubs reported a strong correlation between lower injury incidence rates and team success. ${ }^{7}$ However, measures accounting for both the frequency and severity of injuries (ie, injury burden) are likely to be superior for assessing the impact of injuries on team success, compared with injury incidence rates alone, because injury burden relates more closely to player availability. ${ }^{18}$ Indeed, in an 11-season study of 24 European football teams participating in their countries' highest domestic competition and the UEFA Champions League or Europa League tournaments, a lower injury burden was associated with a higher final domestic league ranking. ${ }^{2}$ Further studies in elite football populations have reported similar correlations between team success (league ranking) and injury burden, ${ }^{9}$ as well as a higher injury incidence rate for matches lost compared to matches won or drawn. ${ }^{10}$ The only study to date to examine the association between injuries and success in Rugby Union teams reported a moderate but nonsignificant correlation $(r=0.31, p=0.2)$ between average days' absence per team and final league position, ${ }^{11}$ although this two-season study may have been underpowered to clearly detect such an association. While the balance of evidence does indicate that a negative association exists between injury measures and success in team sports, such evidence is not abundant, especially with regards to elite Rugby Union populations.

In terms of player welfare, providing evidence of a substantial association between injury measures and team success in Rugby Union may be beneficial for communicating the importance of injury prevention to stakeholders. Accordingly, the aim of this study was to examine the association between injury measures and the success of professional Rugby Union teams.

\section{METHODS}

\section{Study design and setting}

A prospective cohort design was used to record all match and training time-loss injuries associated 
with players in the English Premiership as part of the Professional Rugby Injury Surveillance Project (PRISP). All Premiership teams were required to submit injury and exposure data for PRISP as a constituent of their competition agreement, and were required to meet minimum standards with respect to the timeliness of return and completeness of data. Data collected from the 12 league teams in each of the 7 seasons between 2006/2007 and 2012/2013 were included in the analysis, giving rise to a total of 15 teams due to promotions and relegations during this period.

\section{Participants}

All consenting players that were members of the club's first team squad were eligible for inclusion. The study was approved by the research ethics committee of the academic host institution where the PRISP was based for each season, and written informed consent was obtained from each participant.

\section{Variables}

Injury definition

The definitions and procedures used in this study were consistent with the international consensus statement for epidemiological studies in Rugby Union. ${ }^{12}$ Reported injuries were included in the analysis if they occurred in training or first-team competitive matches (Premiership, National Cup and European competition fixtures), and if they met the $24 \mathrm{~h}$ time-loss injury definition. ${ }^{12}$ All injuries were recorded by medical personnel at each club using a modified Orchard Sports Injury Classification System (OSICS $)^{13}$ and a standardised injury report form. Individual match and grouped training exposure data were reported weekly by strength and conditioning staff using a standard training report form.

\section{Injury measures}

Team injury rates for each season are expressed using injury burden ('overall injury incidence rate $\times$ mean absence per injury', expressed as number of injury days lost per 1000 player hours) in order to account for both the frequency and severity (days lost from competition and practice) ${ }^{12}$ of injuries. As bias may be introduced when combining match and training injury data, due to differences in the ratio of training to match exposure and injury incidence rates between teams, ${ }^{8}$ injury days per teammatch (total team injury time-loss days (match and training)/ number of team-matches) was also included as an independent variable in a separate model. The injury days per team-match variable was included to verify that inferences made using the injury burden variable were accurate.
Team success measures

Two team success measures were used in the analysis: Premiership league points tally and season average Eurorugby Club Ranking (ECR). ${ }^{14}$ The ECR provides an indexed rating of Europe's top teams, and was included to account for team's performances in European competitions. Each week, the ECR system uses the results of all domestic and European ties and awards points for winning or drawing a match, while also making adjustments for factors such as: points conceded and scored, home advantage, strength of opponent, strength of domestic league, importance of the game and recent form. The number of ECR points accumulated by each team is expressed as a percentage of the top-ranked team. Additional team success indicators (final league ranking, points differential and tries scored) are displayed in table 1 for descriptive purposes only.

\section{Statistical methods}

The analyses used in this study were based on the statistical methods employed by Higham et $a l^{15}$ for investigating the association between performance indicators and match outcomes in international Rugby Sevens. All estimations were made using the lme4 package ${ }^{16}$ with R (V.3.0.3, R Foundation for Statistical Computing, Vienna, Austria). Mean values and true betweenteam and within-team SDs for injury and success measures were obtained using a mixed-model reliability analysis. A linear mixed model was then used to estimate the association between the injury and team success measures within each team. Injury measures were included as the linear fixed effect, with the team success measure (league points tally or ECR) as the dependent variable, a random effect for team and season and an interaction effect for injury measure and team. Team squad size (total number of Rugby Football Union registered players) was included in the model to control for its effect. A first-order autoregressive covariance structure was adopted. Alkaike's Information Criterion and the 2 Log Likelihood were used to assess and compare the model's goodness of fit.

The linear mixed model was used to determine the association between injury measures and performance within each team (across the multiple seasons). Between-team effects were analysed to determine how the injury measures of teams that were more successful (on average) over the study period compared to those that were less successful; this was undertaken using averaged values of the injury and team success measures for each team across the seven seasons. All effects were evaluated as the change and difference in team performance associated with a two within-team and between-team SD increase in the injury measures, which represents a change from a typically low to a typically high value. ${ }^{17}$ In addition, Pearson

Table 1 Descriptive summary of team success and injury measures

\begin{tabular}{|c|c|c|c|c|}
\hline & Mean $\pm 90 \% \mathrm{Cl}$ & Observed SD & Within-team SD & Between-team SD \\
\hline \multicolumn{5}{|l|}{ Team success measures } \\
\hline League points tally & $49.6 \pm 6.1$ & 15.4 & 9.1 & 13.0 \\
\hline Final league ranking & $6.9 \pm 2.4$ & 3.5 & 2.5 & 2.6 \\
\hline Points differential & $0.0 \pm 29.5$ & 126.8 & 86.9 & 117.8 \\
\hline Tries scored & $41.9 \pm 4.7$ & 11.8 & 8.1 & 8.1 \\
\hline Eurorugby Club Ranking & $63.8 \pm 7.8$ & 16.0 & 8.8 & 13.6 \\
\hline \multicolumn{5}{|l|}{ Injury measures } \\
\hline Injury burden (days/1000 player-hours) & $188.9 \pm 44.3$ & 77.4 & 67.9 & 20.1 \\
\hline Injury days per team-match & $64.5 \pm 6.0$ & 21.9 & 19.4 & 6.5 \\
\hline
\end{tabular}


correlation coefficients were used to evaluate between-team associations.

Inferences regarding the effect of the injury variables were assessed using the smallest worthwhile difference in team success and magnitude-based inferences. ${ }^{17}$ The smallest worthwhile difference is given by 0.3 of the typical variation in the team success measures between seasons. ${ }^{18}$ This difference was calculated as the SD of the average season-to-season change in each team success measure, multiplied by $0.3 / \sqrt{ } 2 .{ }^{15}$ Using this method, the threshold for smallest worthwhile change in league points tally was calculated to be three points. Throughout the study period, the average points differential between teams finishing in league position 4th versus 5th (play-off qualification) and 6 th versus 7 th (European Cup qualification) was also three points, supporting its use as a practically meaningful points difference. The threshold for smallest worthwhile change in ECR was $2.64 \%$. A correlation of \pm 0.3 (moderate) was adopted as the smallest worthwhile effect for between-team Pearson correlations. ${ }^{19}$ Effects were classified as unclear if the $\pm 90 \%$ confidence limits crossed thresholds for both positive and negative effects by $>5 \%$. Otherwise, the effect was clear and deemed to have the magnitude of the largest observed likelihood value; positive if associated with superior team performance, negative if associated with poorer team performance and trivial if associated with a non-substantial (below the smallest worthwhile change threshold) change or difference in team performance. $^{20}$ This was qualified with a probabilistic term using the following scale: $<0.5 \%$, most unlikely; $0.5-5 \%$, very unlikely; 5-25\%, unlikely; 25-75\%, possible; 75-95\%, likely; $95-99.5 \%$, very likely; $>99.5 \%$, most likely. ${ }^{20}$
RESULTS

Injury, squad size and team success measures

Data from a total of 1462 professional Rugby Union players across 15 teams were included in the analysis. A total of 14 eligible players over the study period did not provide consent, and as such their data were not recorded. In total, 883953 player hours (match, 56 090; training, 827 863) of exposure and 6967 time-loss injuries (match, 4886; training, 2081) were recorded during the study period. This equated to a match injury incidence rate of 87.1 per 1000 player match hours (95\% CI 85.1 to 89.2 ) and a training injury incidence rate of 2.5 per 1000 player training hours (95\% CI 2.4 to 2.6). The overall injury incidence rate was 7.9 injuries per 1000 player hours. The mean severity of all recorded injuries was $24 \pm 41$ days. Mean squad size was $45 \pm 6$ players. Team success measures typically displayed greater variability in differences between teams than changes within teams (table 1). For both injury measures, variability in changes within teams was greater than differences between teams.

\section{Association between injury measures and team success}

The effect of a 2 SD increase in each injury measure (injury burden and injury days per team-match) is shown separately for each of the team success measures (league points tally and ECR) in figure 1. Additional interaction effects between squad size and injury measures were removed from the model as they did not improve model fit and explained no additional variance in team success. Both injury measures displayed clear negative associations with team success (70-100\% likelihood), with the exception of between-team differences for injury days
A - Within-team

- Between-team

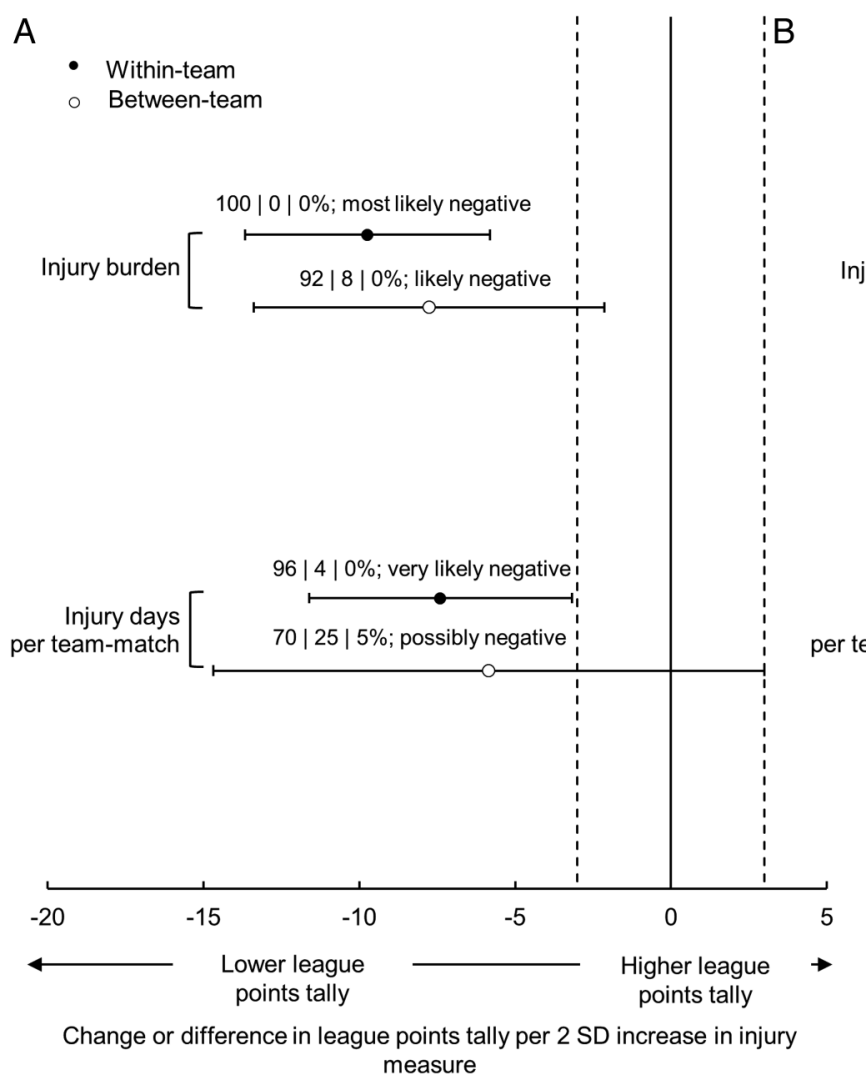

Change or difference in league points tally per 2 SD increase in injury measure

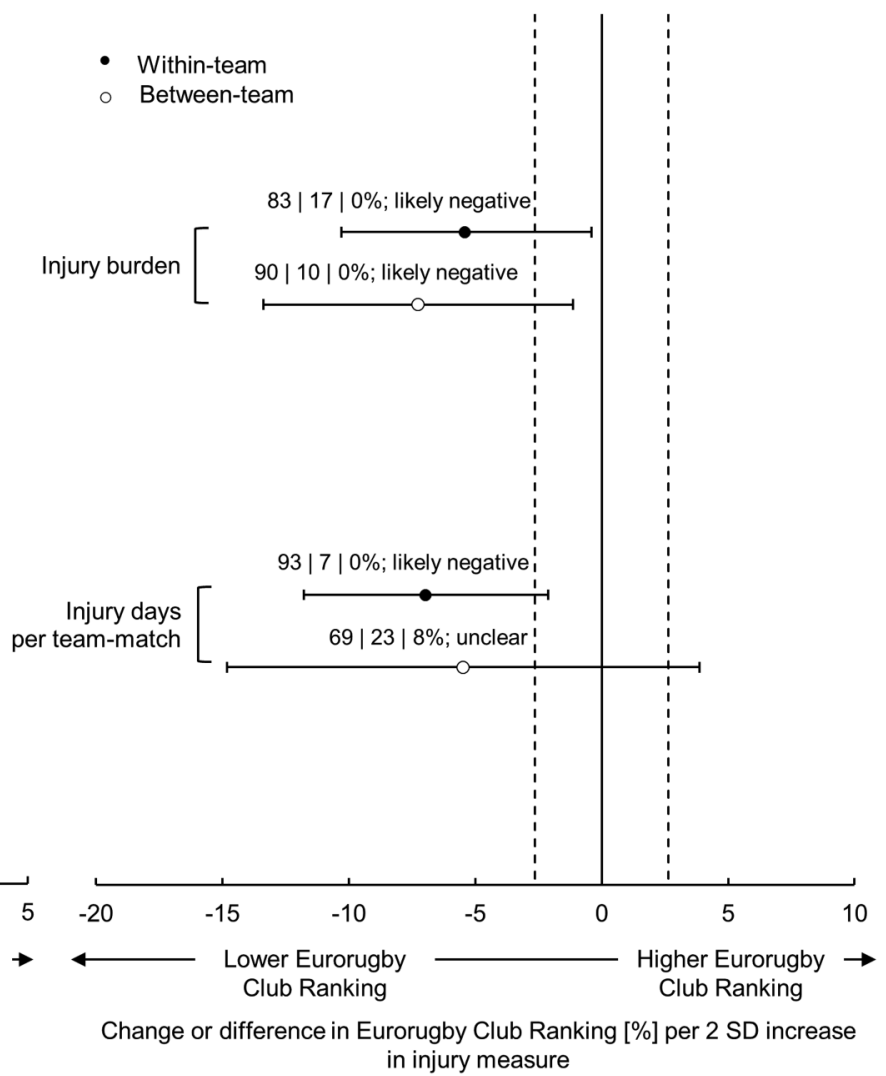

Figure 1 Effect of two SDs of within-team changes and between-team differences of injury measures on (A) league points tally and (B) Eurorugby Club Ranking. Bars are $90 \%$ Cls. Dotted lines represent thresholds for smallest worthwhile difference: (A) \pm 3 league points and (B) $\pm 2.64 \%$. Data labels give \% likelihood that the effect is negative|trivial|positive, and associated qualitative inference. 
per team-match and the ECR success measure, which had an unclear effect. Figure 2 displays the Pearson correlations for team values averaged over all seasons; clear negative associations between the injury and team success measures were observed, with the exception of ECR and injury days per team-match, for which the association was possibly trivial. Both injury measures (injury burden and injury days per teammatch) displayed similar associations with the team success measures.

Based on the average within-team effect, a reduction in injury burden of 42 days per 1000 player hours (90\% CI 30 to 70 ), or a reduction in injury days lost per team-match of 16 days (90\% CI 10 to 36), was associated with the smallest worthwhile change in league points tally ( +3 league points). Similarly, a reduction in injury burden of 66 days per 1000 player hours (90\% CI 34 to 644), or a reduction in injury days lost per teammatch of 15 days ( $90 \%$ CI 9 to 46), was associated with the smallest worthwhile change in ECR $(+2.64 \%)$.
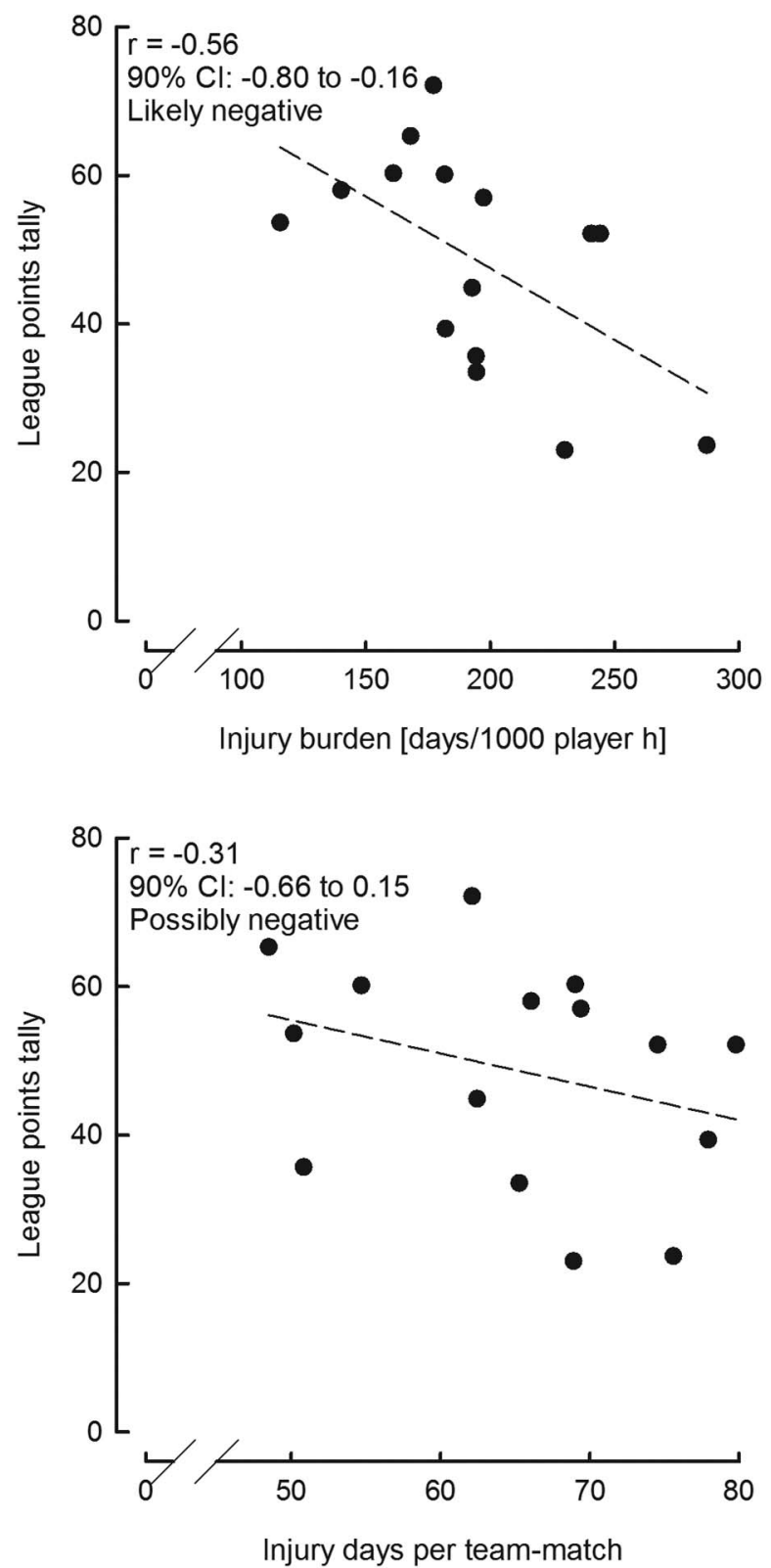

\section{DISCUSSION}

This study sought to establish whether there is an association between injury measures and team success in professional Rugby Union. Both injury measures had clear negative withinteam associations with league points tally and ECR, such that two SD decreases in the injury measures were associated with substantial (worthwhile) improvements in the team success outcomes. Between-team differences in injury measures were also associated with team success measures; teams with low injury measure values typically accumulated more league points and had higher ECR rankings.

\section{Association between injuries and team success}

The results of the current study are in line with the majority of previous studies investigating the association between injuries and performance in elite football (soccer) teams. ${ }^{2} 79$ The mechanisms through which injuries may be associated with team success are likely to be similar between different team sports;
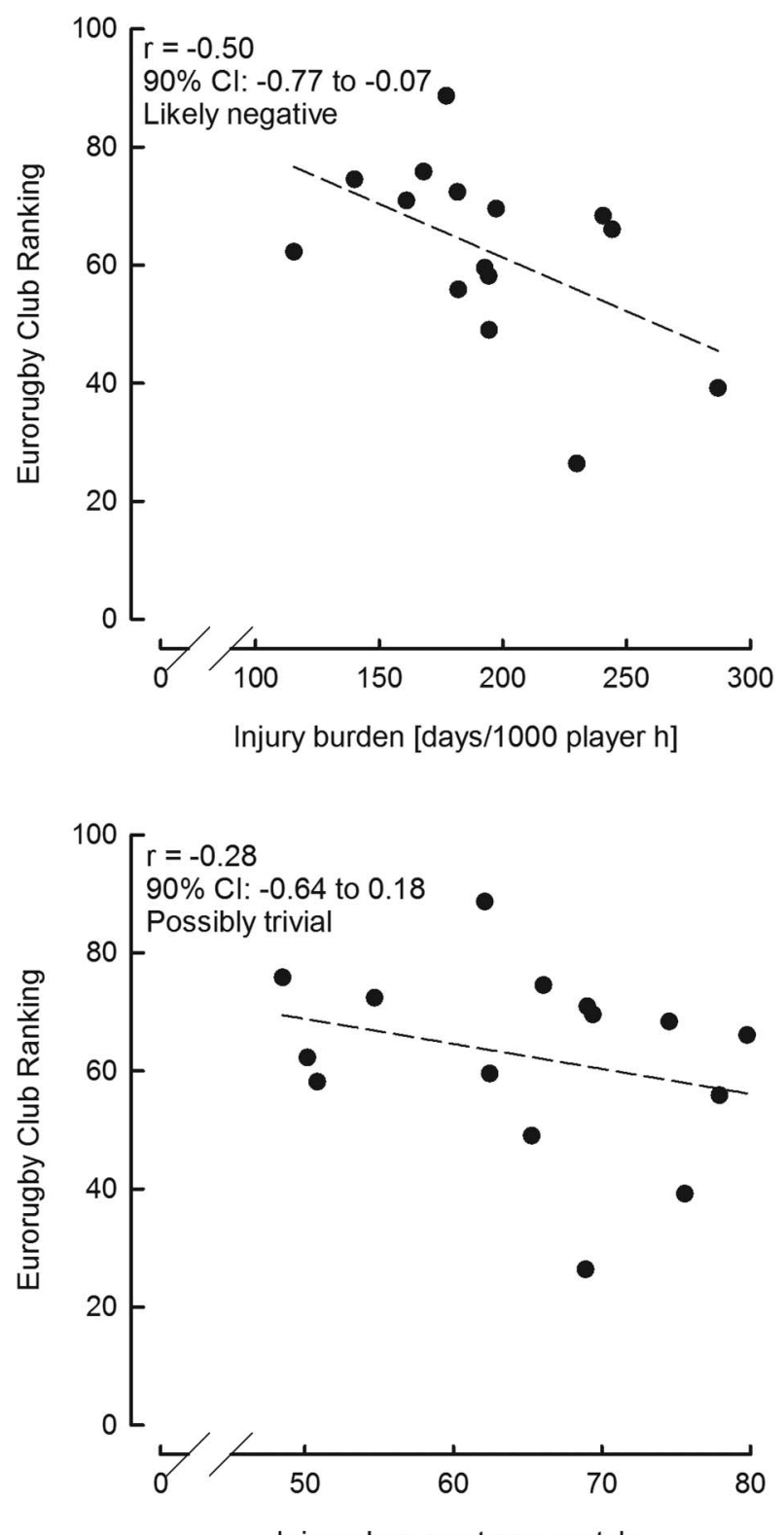

Injury days per team-match

Figure 2 Pearson correlations, $90 \% \mathrm{Cl}$ and qualitative inference for team-averaged values of each injury (injury burden and injury days per team-match) and team success (league points tally and Eurorugby Club Ranking) outcome. Smallest worthwhile effect: $r= \pm 0.3$. 
that is, an inability to select the best players for a given match, disruptions to match preparations during training sessions, and the potential negative psychological effects (such as stress and anxiety) that may be associated with injury events and which may persist when players return from injury. ${ }^{3}$ Injury incidents that occur within a given match are also likely to negatively affect the result of that particular match. ${ }^{10}{ }^{21}$ This may be explained by the fact that the strongest team is typically selected to play, so an injury to any player will weaken the team. Additionally, an injury may require a team to alter their tactical strategy, and may result in players playing out of their favoured position, both of which could reduce the team's chance of winning. ${ }^{10}{ }^{21}$ These findings highlight the potential importance of injury prevention efforts and optimal treatment of injuries for improving team success, in addition to the obvious player welfare considerations.

A within-team change in injury burden of approximately 42 days per 1000 player hours was associated with the smallest worthwhile change in league points tally ( \pm 3 league points). As an illustrative example, this would equate to a typical Premiership team reducing the total number of injuries incurred per season by approximately 13 injuries (in the context of a mean of 83 injuries per team per season during the study period), alongside a two-day reduction in the average severity of all injuries (in the context of a mean injury severity of 24 days during the study period). One possible method that has been suggested for achieving such a reduction in injury burden is to develop and evaluate less conservative return-to-play protocols by implementing more comprehensive rehabilitation strategies for selected injuries (eg, muscle strains). ${ }^{22}$ However, a comprehensive understanding of the risk of subsequent injury and the individual risk factors for early recurrence in this population is required before such a strategy could be recommended. Elsewhere, reductions in injury burden are likely to be best achieved through the targeting of injuries that occur in 'controllable' settings such as set-pieces, training sessions and non-contact injury incidents; on average, 41 injuries per team were sustained in such situations during the 2012/2013 English Premiership season. ${ }^{23}$ The use of psychological interventions (eg, cognitive restructuring and relaxation skills) may also help towards reducing injury burden in this setting. ${ }^{24}$

As causality cannot be directly inferred from these findings, it may be that successful teams incur fewer and/or less severe injuries as a result of being successful. Winning teams are typically involved in fewer tackle situations in elite Rugby Union; $;{ }^{25}$ since the tackle is the most common injury event, ${ }^{26}$ successful teams may have a lower inherent match injury risk. What is more, successful teams may have greater budgets available for medical, rehabilitation and strength and conditioning staff and services. In addition, players in poorly performing teams typically experience a greater degree of anxiety, ${ }^{27}$ which may augment their injury risk. ${ }^{3}{ }^{4}$ It is likely a combination of these factors explains the association between injury measures and team success observed in the current study. Recently developed 'additivenoise methods' may be useful for distinguishing cause from effect in relation to these data, but these methods require further refinement at present. ${ }^{28}$

\section{Methodological considerations}

It should be noted that several potentially important factors were not considered in the current study. For instance, changes in coaching staff and alterations in training/recovery practices could all moderate the association between injury measures and team success, but the effect of these factors was not included in the present analyses. Moreover, no adjustment was made for the relative importance that an injured player had within their team; injuries to a team's best players are likely to have a greater impact on team success than injuries to lesser ranked players. Future investigations of the association between injuries and team success should therefore consider including a weighting factor that accounts for the importance of individual players within a team.

\section{CONCLUSION}

Clear negative associations were found between injury measures and team success, and moderate reductions in injury burden could potentially have a worthwhile effect on competition outcomes for these professional Rugby Union teams. These findings highlight the importance for professional Rugby Union stakeholders to understand the association that exists between injuries and team success, and may be useful when attempting to communicate the value of injury prevention initiatives within this elite sport setting.

\section{What are the findings?}

- Substantial negative associations between injuries and team success were reported for the first time in an elite Rugby Union setting.

- A reduction in injury burden of 42 days $(90 \% \mathrm{Cl} 30$ to 70$)$ per 1000 player hours (22\% of mean injury burden) was associated with the smallest worthwhile change in league points tally ( +3 league points).

\section{How might it impact on clinical practice in the future?}

- These findings highlight the important role that medical, rehabilitation and strength, and conditioning staff have in improving player availability, in order to increase a team's chances of success.

- Knowledge of the negative association between injuries and team success may be useful when attempting to communicate the value of injury prevention initiatives within this elite sport setting (eg, to coaches and administrative staff).

- Coaches, medical, rehabilitation and strength, and conditioning staff should endeavour to work together in an interdisciplinary fashion to prevent injuries.

Twitter Follow Sean Williams at @sw356, Grant Trewartha at @utility_back and Keith Stokes at @drkeithstokes

Acknowledgements The authors would like to acknowledge with considerable gratitude all club medical and strength and conditioning staff for the recording of injury and exposure data throughout the study period.

Contributors SW, KAS and GT conceived and designed the study. MJC, CWF, JHMB and AET were involved in the collection of injury and exposure data. SW analysed the data and wrote the first draft of the manuscript. All authors provided substantial contributions to the redrafting of the manuscript. All authors read and approved the final version of the manuscript.

Funding Funding for this study was provided by the Rugby Football Union, Premier Rugby Limited and University of Bath. 
Competing interests KAS and GT report grants from Rugby Football Union and Premier Rugby Limited during the conduct of the study. AET reports personal fees from the University of Nottingham and the University of Bath during the conduct of the study. SW reports receiving PhD funding (fees and stipend) from Rugby Football Union during the conduct of the study. MJC reports receiving PhD funding (fees and stipend) from Rugby Football Union and Premier Rugby Limited during the conduct of the study. CWF reports grants from Rugby Football Union during the conduct of the study and reports personal fees from World Rugby outside the submitted work. JHMB has nothing to disclose. SPTK is Chief Medical Officer at the Rugby Football Union.

Ethics approval University of Bath.

Provenance and peer review Not commissioned; externally peer reviewed.

\section{REFERENCES}

1 Orchard JW. On the value of team medical staff: can the "Moneyball" approach be applied to injuries in professional football? Br J Sports Med 2009;43:963-5.

2 Hagglund $M$, Walden $M$, Magnusson $H$, et al. Injuries affect team performance negatively in professional football: an 11-year follow-up of the UEFA Champions League injury study. Br J Sports Med 2013;47:738-42.

3 Ivarsson A, Johnson U, Podlog L. Psychological predictors of injury occurrence: a prospective investigation of professional Swedish soccer players. J Sport Rehabil 2013;22:19-26.

4 Lavallee L, Flint F. The relationship of stress, competitive anxiety, mood state, and social support to athletic injury. J Athl Train 1996;31:296-9.

5 Williams $S$, Trewartha $G$, Kemp $S$, et al. A meta-analysis of injuries in senior men's professional Rugby Union. Sports Med 2013;43:1043-55.

6 Dauty M, Collon S. Incidence of injuries in French professional soccer players. Int J Sports Med 2011;32:965-9.

7 Eirale C, Tol JL, Farooq A, et al. Low injury rate strongly correlates with team success in Qatari professional football. Br J Sports Med 2013;47:807-8.

8 Brooks JHM, Fuller CW. The influence of methodological issues on the results and conclusions from epidemiological studies of sports injuries-illustrative examples. Sports Med 2006;36:459-72.

9 Arnason A, Sigurdsson SB, Gudmundsson A, et al. Physical fitness, injuries, and team performance in soccer. Med Sci Sports Exerc 2004;36:278-85.

10 Ekstrand J, Walden M, Hagglund M. Risk for injury when playing in a national football team. Scand J Med Sci Sports 2004;14:34-8.
11 Brooks JH, Fuller CW, Kemp SP, et al. An assessment of training volume in professional rugby union and its impact on the incidence, severity, and nature of match and training injuries. J Sports Sci 2008;26:863-73.

12 Fuller CW, Molloy MG, Bagate C, et al. Consensus statement on injury definitions and data collection procedures for studies of injuries in rugby union. $\mathrm{Br} J$ Sports Med 2007;41:328-31.

13 Orchard J. Orchard Sports Injury Classification System (OSICS). Sports Health 1993;11:39-41.

14 Eurorugby Club Ranking. European Club Rugby Ranking Table 2013 (12 June 2013). http://www.eurorugby.com/modules.php?name=Rdesc

15 Higham DG, Hopkins WG, Pyne DB, et al. Performance indicators related to points scoring and winning in international rugby sevens. J Sports Sci Med 2014;13:358-64.

16 Bates D, Maechler M, Dai B. The Ime4 package 2008 (cited 1 August 2014). http:// cran.r-project.org/web/packages/lme4/lme4.pdf

17 Hopkins W, Marshall S, Batterham A, et al. Progressive statistics for studies in sports medicine and exercise science. Med Sci Sports Exerc 2009;41:3-12.

18 Hopkins WG, Hawley JA, Burke LM. Design and analysis of research on sport performance enhancement. Med Sci Sports Exerc 1999;31:472-85.

19 Hopkins WG. Linear models and effect magnitudes for research, clinical and practical applications. Sportscience 2010;14:49-57.

20 Batterham AM, Hopkins WG. Making meaningful inferences about magnitudes. Int J Sports Physiol Perform 2006;1:50-7.

21 Gabbett TJ. Influence of injuries on team playing performance in Rugby League. J Sci Med Sport 2004;7:340-6.

22 Orchard J, Best TM, Verrall GM. Return to play following muscle strains. Clin J Sport Med 2005;15:436-41.

23 Kemp SPT, Brooks JHM, Cross M, et al. England Professional Rugby Injury Surveillance Project 2012-2013 Season Report 2013 (5 June 2014). http://www.rfu. com/news/2014/february/news-articles/ /media/files/2014/takingpart/injury_audit_ report_2014_.ashx

24 Williams JM, Andersen MB. Psychosocial antecedents of sport injury: review and critique of the stress and injury model'. J App/ Sport Psychol 1998;10:5-25.

25 van Rooyen M, Yasin N, Viljoen W. Characteristics of an 'effective' tackle outcome in Six Nations rugby. Eur J Sport Sci 2014;14:123-9.

26 Fuller CW, Brooks JH, Cancea RJ, et al. Contact events in rugby union and their propensity to cause injury. Br J Sports Med 2007;41:862-7.

27 Maynard IW, Howe BL. Interrelations of trait and state anxiety with game performance of rugby players. Percept Mot Skills 1987;64:599-602.

28 Mooij JM, Peters J, Janzing D, et al. Distinguishing cause from effect using observational data: methods and benchmarks. J Mach Learn Res 2014; In Press arXiv preprint arXiv:1412.3773. 